\title{
Négritude, Universalismus und Avantgarde
}

\author{
Die mosambikanische Literatur und der Premier congrès inter- \\ national des écrivains et artistes noirs in Paris (1956)
}

\begin{abstract}
Alioune Diop bezeichnete in seiner Eröffnungsrede den Premier congrès international des écrivains et artistes noirs im September 1956 als einen Meilenstein für alle Angehörigen afrikanischer Kulturen. Eine ganze Reihe afrikanischer Intellektueller und Schriftsteller hatte sich anlässlich des Kongresses in Paris zusammengefunden, der Welthauptstadt der Avantgarde zur damaligen Zeit. Pablo Picasso hatte das Plakat zur Ankündigung gestaltet. ${ }^{1}$ Unter den Kongressteilnehmern befand sich der junge mosambikanische Student und Autor Marcelino dos Santos. Zwei seiner frühen Gedichte stehen im Mittelpunkt der folgenden Überlegungen zur Dynamik der Begegnungen zwischen unterschiedlichen geistigen Strömungen jener Zeit in Paris. Für die afrikanischen Intellektuellen ging es einerseits darum, sich intern über die Rolle der Literatur für einen panafrikanischen Antikolonialismus sowie für spezifische nationale Kontexte zu verständigen. Andererseits kam es zu einer Auseinandersetzung zwischen afrikanischen und europäischen Avantgarden.
\end{abstract}

Der Schriftstellerkongress in Paris war ein Impuls, auf den weitere Veranstaltungen folgen sollten, wie etwa ein Kongress 1959 in Rom. ${ }^{2} 1956$ war ein weltpolitisch bedeutendes Jahr in einem Jahrzehnt der Umbrüche und Neuordnungen nach dem Ende des Zweiten Weltkrieges: Am 25. Februar hielt Nikita Chruschtschow seine Geheimrede auf dem 20. Parteitag der Kommunistischen Partei der Sowjetunion, die die Verbrechen unter dem drei Jahre zuvor verstorbenen Diktator Josef Stalin offenlegte. Die angestrebte Politik der sogenannten friedlichen Koexistenz war nach der Niederschlagung des Bürgeraufstandes in der DDR am 17. Juni 1953 von der Situation eines atomaren «Gleichgewichts des Schreckens» abgelöst worden. 1955 trat der Warschauer Pakt als Gegenstück zum NATO-Vertrag in Kraft. Unmittelbar nach dem Schriftstellerkongress in Paris kam es im

1 Vgl. das Foto in Présence Africaine 8/10 (Juni-November 1956), S. 9. Vgl. zum Interesse Picassos an afrikanischer Kunst auch die historisch-kritischen Bemerkungen in Achille Mbembe: Kritik der schwarzen Vernunft. Aus dem Französischen von Michael Bischoff. Berlin: Suhrkamp 2017 (Taschenbuch Wissenschaft 2205), S. 85.

2 Vgl. Bennetta Jules-Rosette: Black Paris. The African Writers' Landscape. Urbana/Chicago: University of Illinois Press 1998, S. 51. Für einen konzisen Überblick zur Forschung über den Kongress sowie eine knappe Zusammenfassung der wichtigsten Kongressbeiträge vgl. ebda., S. 52ff.

Ә Open Access. ( 2020 Lucia Weiß, publiziert von De Gruyter. (c) BY-NC-ND Dieses Werk ist lizenziert unter der Creative Commons Attribution-NonCommercial-NoDerivatives 4.0 Lizenz.

https://doi.org/10.1515/9783110679366-013 
Oktober 1956 zum Ungarn-Aufstand, Ende desselben Monats begann die Suezkrise. Frankreich war seit 1954 im Algerienkrieg, die USA seit 1955 im Vietnamkrieg engagiert. Dieser ausschnitthafte Blick auf die weltpolitische Lage der Zeit soll zumindest andeuten, in welch konfliktgeladene Atmosphäre sich der Premier congrès international des écrivains et artistes noirs einschrieb. Die politische Konfiguration spielte eine nicht geringe Rolle in den Debatten, insbesondere im Spannungsverhältnis zwischen den afroamerikanischen und afrikanischen Intellektuellen, ${ }^{3}$ die teilweise der Kommunistischen Partei angehörten. Aimé Césaire, einer der prominentesten Teilnehmer, trat kurz nach dem Kongress aus der Kommunistischen Partei aus. ${ }^{4}$

Das Zusammentreffen der Schriftsteller an der Sorbonne sei, so sagte Alioune Diop damals, in seiner Bedeutung geradezu ebenbürtig mit der Bandung-Konferenz in Indonesien 1955. Dort waren 29 Länder bestrebt gewesen, sich unter der Selbstbezeichnung «Dritte Welt» von den beiden rivalisierenden Parteien des Kalten Krieges abzusetzen:

Ce jour sera marqué d'une pierre blanche. Si depuis la fin de guerre, la rencontre de Bandoeng pour les consciences non européennes l'événement le plus important, je crois pouvoir affirmer que ce premier Congrès mondial des Hommes de Culture noirs représentera pour nos peuples le second événement de cette décade. ${ }^{5}$

Delegationen aus 24 Ländern nahmen an dem Schriftstellerkongress in Paris teil. Aus Mosambik, 1956 noch portugiesische Kolonie, war einzig Marcelino dos Santos dabei. ${ }^{6}$ Der damals 27 -jährige Student wirkte in den intellektuellen Kreisen der afrikanischen Diaspora seit Beginn der 1950er Jahre mit, war als Dichter aber

3 Vgl. Bennetta Jules-Rosette: Black Paris, S. 47, S. 55, S. 57, S. 59.

4 In seinem Brief an den Generalsekretär der Kommunistischen Partei Frankreichs, Maurice Thorez, kritisierte Césaire die ausbleibende Erneuerung der Partei nach der Chruschtschow-Rede, die mangelhafte Politik gegenüber den Kolonialgebieten: «Si le but de toute politique progressiste est de rendre un jour leur liberté aux peuples colonisés, au moins faut-il que l'action quotidienne des partis progressistes n'entre pas en contradiction avec la fin recherchée et ne détruise pas tous les jours les bases mêmes, les bases organisationnelles comme les bases psychologiques de cette future liberté, lesquelles se ramènent à un seul postulat: le droit à l'initiative. Je crois en avoir assez dit pour faire comprendre que ce n'est ni le marxisme ni le communisme que je renie, que c'est l'usage que certains ont fait du marxisme et du communisme que je réprouve. [...] Que la doctrine et le mouvement soient faits pour les hommes, non les hommes pour la doctrine ou pour le mouvement.» Aimé Césaire: Lettre à Maurice Thorez. Paris: Présence Africaine 1956.

5 Alioune Diop: Discours d'ouverture. In: Présence Africaine 8/10 (Juni-November 1956), S. 9-19, hier S. 9.

6 Laut dem Zeitzeugen Mário Pinto de Andrade aus Angola gab es jedoch keine offiziellen Delegationen aus den lusophonen Kolonien: Aus Angola seien zufällig De Andrades Bruder sowie Manuel Lima vor Ort gewesen, aus Mosambik wie erwähnt der in Paris studierende Marcelino dos 
noch nicht prominent in Erscheinung getreten, wie sich sein Weggefährte Mário Pinto de Andrade aus Angola erinnert. ${ }^{7}$ Der vorliegende Beitrag möchte das frühe Werk des Mosambikaners anhand zweier Gedichte genauer untersuchen, die während seiner Zeit im Pariser Exil entstanden sind. ${ }^{8}$ Damit sind sie ein wichtiges, bisher nicht beachtetes Zeugnis für die Herausbildung und das Selbstverständnis portugiesischsprachiger Schriftliteratur mosambikanischer AutorInnen. Dementsprechend soll beleuchtet werden, wie sich Dos Santos' Schreiben im Hinblick auf panafrikanische Diskurse über négritude sowie im Hinblick auf Avantgarde-Praktiken internationaler KünstlerInnen in der französischen Metropole situiert. In der literaturwissenschaftlichen Forschung wird Dos Santos, späteres Gründungsmitglied der mosambikanischen Befreiungsfront (FRELIMO), als Schriftsteller zwar wahrgenommen, jedoch werden seine Texte kaum im Einzelnen betrachtet. In Handbüchern wird meist nur auf seine pamphletistische Dichtung verwiesen, ${ }^{9}$ die sein späteres Schaffen prägte. Im Rahmen des vorliegenden Beitrages soll gezeigt werden, dass zumindest ausgewählte Texte seines frühen Schreibens eine durch-

Santos. Vgl. Michel Laban: Mário Pinto de Andrade: uma entrevista dada a Michel Laban. Übersetzung von Maria Alexandra Dáskalos. Lissabon: João Sá da Costa 1997, S. 134.

7 De Andrade will Dos Santos zu diesem Zeitpunkt noch nicht Schriftsteller nennen und sagt in einem der Gespräche mit Michel Laban über ihn: «já tinha alguns poemas, mas também não tinha obra» («Er hatte schon einige Gedichte, aber noch kein Werk», meine Übersetzung). Ebda. Von Dos Santos sind zwei Pseudonyme bekannt, unter denen er auch veröffentlichte: Kalungano und Lilinho Micaia.

8 Die zeitliche Einordnung seines Schreibens kann nur auf Grundlage von Archivrecherchen nachvollzogen werden, da die kanonisierte Sammlung von Dos Santos Gedichten keine Nachweise über Erstveröffentlichungen und dementsprechend Datierungen führt. Seine gesamten Gedichte sind gesammelt in dem Band Marcelino dos Santos: Canto do amor natural. Maputo: Associação dos Escritores Moçambicanos 1984.

9 «Marcelino dos Santos [...] was at the forefront of militant poets who wrote pamphletary verse that, during the protracted war, served as a didactic instrument as well as a road to political mobilization. Not noted for its aestheticism, the poetry of this generation is a valuable historical document tracing the ideological concerns of the liberation movement.» Roland Greene/Stephen Cushman u.a. (Hg.): The Princeton Encyclopedia of Poetry and Poetics. Princeton: Princeton University Press ${ }^{4}$ 2012, S. 18. Auch in dem von Patrick Chabal verantworteten Überblickswerk The Postcolonial Literature of Lusophone Africa, das bis heute als Standardwerk für die portugiesischsprachigen Literaturen des afrikanischen Kontinents gilt, findet sich eine ähnliche Einordnung. Chabal zählt Dos Santos in seiner Einführung zu Mosambik zur Kategorie «Nationalist and revolutionary literatures, betont jedoch den arbiträren Charakter diese Schemas und ergänzt: «O [o]ur 〈labelling〉 [...] is not in itself a value judgement on the quality of writing.» Patrick Chabal: The Postcolonial Literature of Lusophone Africa. Evanston: Northwestern University Press 1996 (African Studies), S. 37. Vgl. zur Diskussion um Marcelino dos Santos als literarische Figur auch Fátima Mendonça: O entrelugar da escrita: entre Marcelino ideólogo e Kalungano poeta. In: Dies.: Literatura Moçambicana - as dobras da escrita. Maputo: Ndjira 2011, S. 97-108. 
gearbeitete Ästhetik aufweisen, die mit dem zeithistorischen Anliegen des antikolonialen Befreiungskampfes verknüpft ist und auf ein globales panafrikanisches Imaginarium zurückgreift.

Die ausgewählten Gedichte veranschaulichen verschiedene Aspekte des Schreibens von Dos Santos: Ein Text orientiert sich stärker an sozialistischrealistischer beziehungsweise neorealistischer Ästhetik ${ }^{10}$, der andere ist sprachlich komplexer und arbeitet mit zahlreichen intertextuellen Bezügen. Gemeinsam ist beiden Gedichten - deren Entstehung in die Frühphase der mosambikanischen Unabhängigkeitsbestrebungen fällt, wie Archivrecherchen belegen - ein metapoetisches und damit auch autoreflexives Moment. Die Lyrik bringt demnach nicht nur Hoffnung, sondern sie ist auch ein Akt der positiven Veränderung und der Freiheit. Der Aufenthalt in Paris bot vielen afrikanische AutorInnen den notwendigen Freiraum, solche Ideen zu entwickeln.

In den 1950er-Jahren war Paris ${ }^{11}$ ein geistiges Zentrum für die Unabhängigkeitsbewegungen der portugiesischen Kolonien, die sich erst Mitte der 1970erJahre von der europäischen Herrschaft befreien konnten. ${ }^{12}$ De Andrade begab sich 1954 nach Paris, weil er sich in Lissabon vor der portugiesischen Geheimpolizei (PIDE) nicht sicher wähnte. ${ }^{13}$ Dort kreuzten sich die Wege vieler junger AfrikanerInnen, die später wichtige Rollen im literarischen und politischen Diskurs ihrer Herkunftsländer einnehmen sollten. In der Studentenstadt verbrachte De Andrade, der alsbald für Présence Africaine arbeiten sollte, viel Zeit mit Dos Santos:

Paris para mim, era a minha grande aventura, era a grande aventura intelectual. Encontreime logo com os meus amigos que moravam na Cidade Universitária - Marcelino dos Santos e Aquino de Bragança -, com quem estava em contacto. Marcelino dos Santos escrevia-nós muitas vezes para Lisboa. Levou-me a casa de Alioune Diop, e nós falámos imediatamente da possibilidade de emprego. ${ }^{14}$

10 Vgl. den Beitrag von Diana Gomes Ascenso im vorliegenden Band.

11 Zur Rolle Paris für afrikanische und afroamerikanische kulturelle Entwicklungen vgl. u.a. Bennetta Jules-Rosette: Black Paris sowie für den Beginn des 20. Jahrhunderts etwa Petrine Archer-Straw: Avant-Garde Paris and Black Culture in the 1920s. New York u.a.: Thames and Hudson 2000. Über eine der prominentesten Künstlerinnen, Josephine Baker, liegen außerdem zahlreiche Arbeiten vor. Vgl. etwa Phyllis Rose: Jazz Cleopatra: Josephine Baker in her Time. New York: Vintage 1991.

12 Malyn Newitt weist darauf hin, dass es unter den Exil-MosambikanerInnen in Lissabon und Paris zwar einige politische Aktivität gegeben habe, aber dass sich ihr Einfluss für die Unabhängigkeitsbewegung in Grenzen gehalten habe. Vielmehr seien Exil-MosambikanerInnen in Tansania, Malawi und dem damaligen Rhodesien mit ihren Bestrebungen letztlich erfolgreicher gewesen. Vgl. Malyn Newitt: A short history of Mozambique, Hurst and Company 2017, S. 138.

13 Vgl. Michel Laban: Uma entrevista, S. 108f.

14 Ebda., S. 109. 
Paris erlaubte eine ungleich größere politische und künstlerische Freiheit, als unter den repressiven Verhältnissen im Estado Novo seinerzeit gegeben:

[...] poderíamos ter uma Présence Africaine em Lisboa, por que não? Mas não tivemos este orgão, evidentemente por circunstâncias claras: o regime político português. O que não pudemos fazer em Lisboa, queríamos realizar em Paris, com um homem como Alioune Diop. ${ }^{15}$

Das Bestreben nach der nationalen Unabhängigkeit Mosambiks war in besonders enger Weise mit dem Bemühen um eine Nationalliteratur ${ }^{16}$ verbunden, das sich im Kraftfeld von transnationalen Strömungen wie der négritude und dem Surrealismus artikulierte. Ein Höhepunkt des panafrikanischen intellektuellen Diskurses war der Premier congrès des écrivains noirs 1956. ${ }^{17}$ Alioune Diop aus Senegal, der 1947 die Zeitschrift ${ }^{18}$ und 1949 das gleichnamige Verlagshaus Présence Africaine gegründet hatte, setzte den Impuls. ${ }^{19}$ Die Liste der Teilnehmenden des Zusammentreffens vom 19. bis 22. September 1956 liest sich wie das Who-isWho afrikanischer Intellektueller der Zeit: Aimé Césaire, Léopold Sédar Senghor, Frantz Fanon, Richard Wright, René Depestre, Édouard Glissant, Amadou Hampaté Bâ, Jacques Rabemananjara, Cheikh Anta Diop um nur einige der prominentesten zu nennen. ${ }^{20}$ Im Nachdenken über die Bedeutung von négritude, dem Verhältnis von politischer Unabhängigkeit und nationaler Literatur sowie dem Engagement der Intellektuellen wurde hart um einzelne Aspekte gerungen: Dabei ging es etwa um die Frage, ob Gewalt im antikolonialen Protest zu legitimieren sei und inwiefern sich eine eigenständige literarische Tradition in Ländern mit langandauernder kolonialer Unterdrückung herausbilden könne. ${ }^{21}$ In Paris verhandel-

15 Ebda., S. 110. «Wir könnten eine Présence Africaine in Lissabon haben, warum nicht? Aber wir hatten dieses Publikationsorgan nicht, natürlich wegen der offenkundigen Umstände: dem politischen Regime in Portugal. Was wir nicht in Lissabon in die Tat umsetzten konnten, wollten wir in Paris tun, mit einem Mann wie Alioune Diop.» (meine Übersetzung)

16 Aimé Césaire und René Depestre trugen einen Disput zur Frage nach „nationaler Poesie“ aus. Vgl. als Überblick dazu Bennetta Jules-Rosette: Black Paris, S. $208 \mathrm{ff}$.

17 Vgl. Présence Africaine 8/10.

18 Der Literaturkritiker und Schriftsteller Simon Njami sagt über die Zeitschrift Présence Africaine: «it will represent, until the 1960s, the necessary crossroads for all black movements». Bennetta Jules-Rosette: Black Paris, S. xii.

19 Vgl. zu den Zielen von Présence Africaine die Ausführungen Diops anlässlich der Gründung: Alioune Diop: Niam n'goura ou les raisons d'être de Présence Africaine. In: Présence Africaine 1 (November-Dezember 1947), S. 7-14.

20 Vgl. für die vollständige Übersicht Présence Africaine 8/10, S. 407.

21 «Colonial discourses such as négritude encompass not only monolithic ideas, but also the speech of interacting human beings, espousing different points of view. One need only reexamine the debates sponsored by Présence Africaine on the role of violence in protest (Fanon vs. Diop), national poetry (Césaire vs. Depestre), and colonization and tradition (Wright vs. Cesaire and 
ten afrikanische Intellektuelle und KünstlerInnen somit auf zwei Ebenen, die allerdings miteinander verwoben waren. Auf der einen Seite stand eine panafrikanische Debatte über ein gemeinsames Selbstverständnis, das sich in zentralen Konzepten wie der négritude artikulierte. Auf der anderen Seite stand das spannungsreiche Verhältnis zwischen afrikanischen Avantgarden - im Sinne einer pointiert in Kunst und Literatur zum Ausdruck gebrachten Selbstbehauptung und europäischen Avantgarden. ${ }^{22}$

Um das Verhältnis zwischen négritude-DichterInnen und europäischen AvantgardIstinnen präziser zu fassen, sind die Ausführungen von Achille Mbembe hilfreich: Die SurrealistInnen, allen voran André Breton, erhielten über ihre Kontakte zu libertären und trotzkistischen AktivistInnen Zugang zu afrikanischen, lateinamerikanischen und karibischen KünstlerInnen, die antikolonial eingestellt waren. ${ }^{23}$ Dadurch schöpften sie Inspiration für ihr eigenes Schaffen. Der kamerunesische Historiker Mbembe diskutiert kritisch das Interesse an Afrika von Seiten europäischer KünstlerInnen zu jener Zeit. Dabei geht er insbesondere auf Bretons Aussagen über die «Affinitäten zwischen dem so genannten primitiven Denken und dem surrealistischen Denken» ${ }^{24}$ ein:

Das «schwarze Vorbild» öffnet nach dieser Vorstellung den Weg zu einer neuen Art des Schreibens. Es geht auch darum, den wilden Charakter der Sprache wiederzuentdecken und das Wort wieder zum Leben zu erwecken, da die Fülle der Sprache sich erst in der Plastizität des Idioms erschließe. ${ }^{25}$

Mbembe legt dar, welche Ambivalenzen in der antikolonialen Kritik von Seiten der EuropäerInnen steckten ${ }^{26}$ und schließt: «Sie hat nicht ausreichend Abstand

Fanon) to acquire a sense of the contested terrains surrounding négritude discourse.» Bennetta Jules-Rosette: Black Paris, S. 88. René Depestre distanzierte sich später von der négritude, u.a mit seinem Buch Bonjour et adieu à la négritude. Paris: Robert Laffont 1980. Vgl. ebda., S. 93-96.

22 Ich spreche bewusst von Avantgarden im Plural, um den vielfältigen Artikulationen von Avantgarde, die sowohl von Seiten afrikanischer als auch europäischer und weiterer KünstlerInnen gemacht wurden, Rechnung zu tragen. Vgl. dafür das Erkenntnissinteresse und die weiteren Beiträge des vorliegenden Bandes.

23 Vgl. Achille Mbembe: Kritik der schwarzen Vernunft, S. 88.

24 Ebda., S. 87.

25 Ebda.

26 «Diese ästhetische, mit Anarchismus und Avantgardismus vermischte Kritik ist nicht frei von Ambivalenzen. Einerseits stützt sie sich weitgehend auf die damals modischen Überlegungen zur 〈afrikanischen Seele» und das angebliche Wesen des «Schwarzen〉. Diese spekulativen Konstruktionen sind das unmittelbare Erbe der westlichen Ethnologie und der Geschichtsphilosophien, die die zweite Hälfte des 19. Jahrhunderts beherrschen.» Mbembe führt u.a. Lévy-Bruhls Arbeiten an, die zwischen einer «westlich-rationalen〉 und «afrikanisch-wilden〉 Mentalität unterscheiden wol- 
gewonnen von der Vorstellung, wonach das «schwarze Blut eine wesentliche Rolle im Erwachen der Phantasie und des künstlerischen Genies spielt.» ${ }^{27}$ Allerdings, so Mbembe weiter, lag im Rückgriff auf bestimmte koloniale Klischees und Mythen auch ein Berührungspunkt mit der négritude, die diese ins Gegenteil wenden wollte. ${ }^{28}$ Vor dem Hintergrund dieser Einsichten stellt sich die Frage, inwiefern das Verhältnis von DichterInnen rund um die négritude (in ihren verschiedenen Formen) und AvantgardIstinnen nicht differenzierter perspektiviert werden müsste, als es gemeinhin, etwa bei Lylian Kesteloot, der Fall ist. ${ }^{29}$ Ein komparatistischer Zugriff erscheint für dieses Unterfangen unbedingt nötig, um die literaturhistorischen Dynamiken genauer konturieren zu können, wie dieser Beitrag zu zeigen versucht. Analytischer Fluchtpunkt ist die Überlegung, inwiefern sich die Diskussion über Avantgarden Anfang bis Mitte des 20. Jahrhunderts, mit Paris als Zentrum, um weltliterarische Dimensionen erweitern lässt.

Dies soll hier mittels der Analyse zweier ausgewählter Gedichte von Marcelino dos Santos aus den 1950er Jahren geschehen. Eines davon entstand in Paris. Der 1929 geborene Marcelino dos Santos verbrachte wie viele privilegierte AfrikanerInnen die Studienzeit in Europa. ${ }^{30}$ In Lissabon arbeitete er ab 1950 eng mit der Casa dos Estudantes do Império (CEI) zusammen. ${ }^{31}$ Seine Gedichte erschienen in deren Zeitschrift Mensagem ${ }^{32}$ und später in zwei Anthologien der CEI. ${ }^{33}$ Aus Sorge um Verfolgungen durch die portugiesische Geheimpolizei PIDE ging Dos

len. «Andererseits hat diese ästhetische Kritik am Kolonialismus nicht vollständig mit dem Mythos der Existenz «überlegener Völker» gebrochen [...].» Ebda., S. 88.

27 Ebda., S. 89.

28 Vgl. ebda., S. $90 f$.

29 Vgl. Lylian Kesteloot: Césaire et Senghor. Un pont sur l'Atlantique. Paris: L'Harmattan 2006, S. 27-43.

30 «Educational opportunities in Mozambique were limited and this was to be a crucial factor in the development of nationalist movements.» Malyn Newitt: A short history of Mozambique, S. 136. Vgl. dazu auch Andreas Eckert: Universitäten und die Politik des Exils. Afrikanische Studenten und anti-koloniale Politik in Europa, 1900-1960. In: Rüdiger vom Bruch u. a. (Hg.): Jahrbuch für Universitätsgeschichte 7. Stuttgart: Franz Steiner 2004, S. 129-145.

31 Vgl. Inocência Mata: A Casa dos Estudantes do Império e o lugar da literatura na consciencialização política. Lissabon: União das Cidades Capitais de Língua Portuguesa 22015.

32 Nicht zu verwechseln mit der gleichnamigen Zeitschrift aus Angola.

33 Vgl. Maria do Rosário Rosinha (Hg.): Antologias de poesia da Casa dos Estudantes do Império 1951-1963. Bd. 2. Mosambik/Lissabon: União das Cidades Capitais de Língua Portuguesa 2014. Außerdem enthalten die zwei thematisch organisierten Anthologien, die Mário Pinto de Andrade herausgegeben hat, seine Gedichte. Vgl. Mário Pinto de Andrade (Hg.): Antologia temática de poesia africana 1. Na nojte grávida de punhais. Lissabon: Sá da Costa 1975 und Ders. (Hg.): Antologia temática de poesia africana 2. O canto armado. Lissabon: Sá da Costa 1979. 
Santos 1951 nach Paris. ${ }^{34}$ Der mosambikanische Schriftsteller und spätere Politiker war in seinen jungen Jahren also zunächst ein privilegierter $«$ Nomade ${ }^{35}$ und wandelte sich dann aufgrund des politischen Drucks zum Exilierten.

Wann und wo genau Dos Santos seine Gedichte schrieb und veröffentlichte, ist zwar gemeinhin nur schwer nachzuvollziehen, weil es an philologischer Aufarbeitung seines Schreibens fehlt. ${ }^{36}$ Jedoch ermöglicht der markante Fund in der mosambikanischen Zeitschrift Itinerário in einer Ausgabe von 1955, auf den Stefan Helgesson hingewiesen hat, ${ }^{37}$ in einem Fall eine präzise Situierung: Dos Santos' Gedicht Aqui nascemos ist dort mit Orts- und Zeitangabe abgedruckt: Paris, 24. Juni $1955 .^{38}$ Dadurch wird die weltumspannende, konkrete wie geistige Konfiguration deutlich, die die Entwicklung der portugiesischsprachigen mosambikanischen Schriftliteratur in dieser Zeit markierte. Insofern scheint ein historisch rückgebundenes close reading dieses Textes besonders geeignet, der Verflechtung panafrikanischer und europäisch-afrikanischer Avantgardepraktiken nachzuspüren.

34 Vgl. dazu auch Malyn Newitt: A short history of Mozambique, S. 137.

$35 \mathrm{Zu}$ einer kritischen Auseinandersetzung mit dem Begriff des Nomaden und dessen Dimensionen in aktuellen Theoriediskussionen vgl. Anna Lipphardt: Der Nomade als Theoriefigur, empirische Anrufung und Lifestyle-Emblem. Auf Spurensuche im Globalen Norden. In: Aus Politik und Zeitgeschichte 65 (Bundeszentrale für Poltische Bildung), 26-27 (2015), S. 32-38.

36 Siehe Marcelino dos Santos: Canto do amor natural.

37 Vgl. Stefan Helgesson: In search of newness. In: Scrutiny2. Issues in English Studies in Southern Africa 10, 2 (2005), S. 15-23, hier S. 21.

Helgesson spricht an anderer Stelle von einer ironischen Konfiguration angesichts des Gegensatzes zwischen Wunsch und Wirklichkeit: «These lines are steeped in the irony of wanting to create an sauthentically Mozambican literature within a transnational discursive context. While it employs a number of rhetorical techniques to suggest immediate unity between poetic subject and land (the deictic shere, the insistent rhythm of anaphoric repetition-see the original), the poem is dated 〈Paris, 24 June 1955〉. Word and world, body and word are separated, the mobility and disembodiedness of print are aptly demonstrated. The poem's performance of national authenticity ought therefore to be read metaphorically, in terms of a secondary reference not only to the intentionality of the poetic subject, but even more to the editorial legacy of the waning Itinerário. Far more committed to the development of modernist, Black Atlantic and African discourses than has generally been acknowledged (if at all) by researchers in the field, it set a precedent for subsequent literary activities in journals such as A Voz de Moçambique (1961-1975) and Caliban (1971-1972).» Stefan Helgesson: Shifting Fields: Imagining Literary Renewal in Itinerário and Drum. In: Research in African Literatures 38, 2 (Sommer 2007), S. 206-226, hier S. 215.

38 Diese Orts- und Zeitmarke findet sich nach meinen Recherchen in der Portugiesischen Nationalbibliothek in Lissabon nur im Originalabdruck. Sie fehlt bei jeglichen Wiederabdrucken in anderen Zeitschriften oder Anthologien, wie etwa in Marcelino dos Santos: Canto do amor natural, S. 13-16. 


\begin{tabular}{|c|c|}
\hline Aqui nascemos.... ${ }^{39}$ & Hier wurden wir geboren... ${ }^{40}$ \\
\hline Ao Meu Irmão & An meinen Bruder \\
\hline I & I \\
\hline A terra onde nascemos & Die Erde auf der wir geboren wurden \\
\hline Vem de longe & Kommt von weit her \\
\hline com o tempo & mit der Zeit \\
\hline Nossos avós & Unsere Großeltern \\
\hline nasceram & wurden geboren \\
\hline e viveram nesta terra & und lebten auf dieser Erde \\
\hline e como ervas de fina seiva & und wie Kräuter von dünnem Lebenssaft \\
\hline foram veias em corpo longo & waren sie Venen eines langen Körpers \\
\hline fluído rubro perfume terrestre & feuerrote Flüssigkeit erdiger Duft \\
\hline Árvores e granitos erguidos & Hoch aufgerichtete Bäume und Granitfelsen \\
\hline seus braços & ihre Arme \\
\hline abraçaram a terra & umschlangen die Erde \\
\hline no trabalho quotidiano & in der täglichen Arbeit \\
\hline e esculpindo as pedras férteis & und während sie die fruchtbaren Steine \\
\hline do mundo a começar & einer aufkommenden Welt behauten \\
\hline em cores iniciaram & begannen sie in Farben \\
\hline 0 grande desenho da vida & das große Bild des Lebens \\
\hline
\end{tabular}

39 Zitiert nach der Erstveröffentlichung in der Zeitschrift Itinerário. Vgl. Kalungano: Aqui nascemos. In: Itinerário 149 (Juli/August 1955), S. 13. Im Rahmen der Gedichtanalyse beginne ich die Verszählung vor der ersten Strophe, bei der vorangestellten Widmung. Die kanonisierte Weitergabe des Gedichtes in Marcelino dos Santos: Aqui nascemos. In: Ders.: Canto do amor natural, ab S. 13-15 unterscheidet sich in einigen Details: Es fehlen die Widmung «Ao Meu Irmão» sowie die Orts- und Zeitangabe, weiterhin unterscheidet die Ausgabe nicht, welche Gedichte unter welchem Pseudonym veröffentlicht wurden. Außerdem gibt es folgende Abweichungen in Groß- und Kleinschreibung bei den Versanfängen: Strophe I, 2, 17 dort jeweils klein sowie Strophe III, 10, 12. Außerdem ist die Kopplung einzelner Verse in den Strophen unterschiedlich: In der Anthologie werden abweichend Strophe III, 1-5 gekoppelt sowie III, 8-11. In Strophe III, 2 wird orthographisch abweichend «xirico» geschrieben.

40 Um die Gedichtanalysen besser nachvollziehen zu können, sind beide Gedichte mitsamt einer von mir angefertigten deutschen Übertragung in dem vorliegenden Beitrag eingefügt. Ich danke Diana Gomes Ascenso und Sophie Bornscheuer für die Hilfe bei der Übersetzung aus dem Portugiesischen. Etwaige Fehler oder Ungenauigkeiten liegen allein in meiner Verantwortung. 


\begin{tabular}{|c|c|}
\hline II & II \\
\hline E foi também & Und es war auch \\
\hline aqui & hier \\
\hline que eu e tu nascemos & wo ich und Du geboren wurden \\
\hline $\begin{array}{l}\text { Terra quente } \\
\text { de sol nascente }\end{array}$ & $\begin{array}{l}\text { Heiße Erde } \\
\text { aufgehender Sonne }\end{array}$ \\
\hline $\begin{array}{l}\text { Terra verde } \\
\text { de campos plenos }\end{array}$ & $\begin{array}{l}\text { Grüne Erde } \\
\text { üppiger Felder }\end{array}$ \\
\hline $\begin{array}{l}\text { Terra meiga } \\
\text { de colo largo }\end{array}$ & $\begin{array}{l}\text { Sanfte Erde } \\
\text { großen Schoßes }\end{array}$ \\
\hline foi a nós & wir waren es \\
\hline que se entregou & denen sie sich hingab \\
\hline cheia de vida & voll von Leben \\
\hline e amorosa ânsia & und liebevoller Ängstlichkeit \\
\hline III & III \\
\hline $\begin{array}{l}\text { Crescemos embalados } \\
\text { no canto do chirico }\end{array}$ & $\begin{array}{l}\text { Wir wuchsen eingehüllt auf } \\
\text { vom Gesang des Chirico }\end{array}$ \\
\hline e brotando assim na planície humana & $\begin{array}{l}\text { und wie wir derart aufkeimten in der Ebene des } \\
\text { Menschlichen }\end{array}$ \\
\hline tão fundo impulso germinou & spross ein so tiefer Impuls \\
\hline ondas fecundas de cristal & fruchtbare kristallklare Wellen \\
\hline $\begin{array}{l}\text { E quando o vento } \\
\text { vergasta o firmamento }\end{array}$ & $\begin{array}{l}\text { Und wenn der Wind } \\
\text { den Himmel verwüstet }\end{array}$ \\
\hline $\begin{array}{l}\text { e a espada cai } \\
\text { e rasga os corpos }\end{array}$ & $\begin{array}{l}\text { und das Schwert niederfällt } \\
\text { und die Körper spaltet }\end{array}$ \\
\hline $\begin{array}{l}\text { O horror tinge } \\
\text { a face crua }\end{array}$ & $\begin{array}{l}\text { Färbt die Grausamkeit } \\
\text { das rohe Gesicht }\end{array}$ \\
\hline O nosso amor não treme & Unsere Liebe erzittert nicht \\
\hline $\begin{array}{l}\text { Esta é a terra } \\
\text { onde nascemos }\end{array}$ & $\begin{array}{l}\text { Dies ist die Erde } \\
\text { auf der wir geboren wurden }\end{array}$ \\
\hline seu sofrer & ihr Leiden \\
\hline é nossa dor & ist unser Schmerz \\
\hline e a nuvem fel de agora & und die bittere Wolke des Jetzt \\
\hline é momento doloroso & ist ein schmerzhafter Moment \\
\hline que a chuva há de secar & den der Regen trocknen muss \\
\hline
\end{tabular}




\section{IV}

Nossa terra é de esperança

aberta ao franco amplexo

Na esteira dos passos dados

vão brilhando círculos livres

e como irmãos mais novos

de um século mais velho

vamos levando em largas mãos

a herança dos nossos avós

e com folhas do coração

continuar a obra humana

o grande desenho da vida.

Paris, 24 de Junho de 1955

\section{IV}

Unsere Erde ist voll von Hoffnung

offen für die ehrliche Umarmung

In der Spur der gemachten Schritte

scheinen fortwährend freie Kreise auf

und wie jüngere Geschwister

eines älteren Jahrhunderts

werden wir mit vollen Händen

das Erbe unserer Großeltern mitnehmen

und mit Blättern des Herzens

das menschliche Werk weiterführen

das große Bild des Lebens.

Paris, 24. Juni 1955

KALUNGANO

KALUNGANO

Das Gedicht Aqui nascemos umfasst vier Strophen, die sich mit der Zugehörigkeit eines lyrischen Wirs beschäftigen. Die zentrale und in Texten zeitgenössischer DichterInnen - wie Noémia de Sousa, Alda Espírito Santo, José Craveirinha, Agostinho Neto - häufig präsente Metapher macht die Erde zu einer Art Urmutter. Die erste Strophe etabliert eine räumliche hic-Deixis: Die Ortsbestimmung ‘aqui des Titels wird im sechsten Vers implizit wieder aufgegriffen mit dem Ausdruck «nesta terra». Damit verortet sich die Sprecherinstanz in der Nähe dieser «terra〉, wohingegen die zeitliche Bestimmung mit den durchgehend im pretérito perfeito simples gehaltenen Verben eine Distanz aufweist. Die Zugehörigkeit des kollektiven lyrischen Subjekts begründet sich indes nicht durch bloße Geburt: Eine innige Beziehung schafft erst die über Generationen hinweg angelegte Kultivierung der Erde, die im Gedicht durch ein Polyptoton nachdrücklich als emotionale Bindung kodiert wird: «seus braços [dos avós] / abraçaram a terra / no trabalho quotidiano» (V. 12-14).

Die zweite Strophe bestimmt den Sehnsuchtsort der Erde in drei parallel aufgebauten Zweizweilern mit jeweils vier beziehungsweise fünf Silben. Die rhythmisch regelmäßige Gestaltung lässt das Trikolon wie einen Lobgesang erklingen. Dazu trägt auch die elliptische Form der Verse bei, die nur an dieser Stelle des Gedichtes vorkommt. Sie bringt den Sprachfluss des Gedichtes zum Innehalten: «Terra quente / de sol nascente / Terra verde / de campos plenos / Terra meiga / de colo largo» (V. 22-27). Wenn von ihrem Schoß in Vers 27 die Rede ist, wird «terra` als Frau beziehungsweise Mutter personifiziert. 
In der dritten Strophe wechselt die verbale Zeit ins Präsens. Das lyrische Subjekt versichert seine unerschütterliche Liebe angesichts natürlicher sowie menschlicher Gewalt (vgl. V. 38-43) und bestimmt die Gegenwart seines Sprechens als eine schmerzvolle (vgl. V. 49-51). Die letzte Strophe evoziert Hoffnung und schließt mit einer wiederaufgegriffenen Formulierung an die erste Strophe an: «e com folhas do coração / continuar a obra humana / o grande desenho da vida.» (V. 61-63). Während 〈o grande desenho da vida〉 zu Beginn des Gedichtes in einer romantisierenden Perspektive auf die Landwirtschaft vornehmlich mit der täglichen Arbeit verbunden ist, steht es zum Schluss des Gedichtes explizit im Kontext des Schreibens. Das menschliche Werk soll «com folhas de coração〉 weitergeführt werden, so heißt es. In dieser selbstreflexiven Geste wird der Dichtung die Aufgabe zugewiesen, die Zugehörigkeit des lyrischen Subjektes zu artikulieren - verstanden als nicht abreißendes Erinnern an die «herança dos nossos ávos».

Dieser frühe Text Dos Santos' imaginiert eine vornehmlich emotional konturierte Topographie. Indem der Text die Erde zur Mutter stilisiert, wird sie zu einem nostalgischen, zeitlos gültigen Inbegriff von Zugehörigkeit. Das lyrische Subjekt begründet seine Liebe zur Erde aber als eine historisch gewachsene, nicht als eine unumstößlich biologisch gegebene - was mit Blick auf die Metapher der Mutter durchaus möglich wäre. Das Entscheidende ist die Beziehung, die sich erst durch die Bewirtschaftung und das vor Ort gelebte Leben konstituiert. Diese Zugehörigkeit ist positiv bestimmt und nicht in Abgrenzung $\mathrm{zu}$ anderen: An keiner Stelle des Gedichtes wird «a terra〉 $\mathrm{zu}$ «a nossa terra〉, was einen ausschließlichen Besitzanspruch markieren würde.

Es ist indes der Zusatz, der auf die spannungsreiche Position des Autors und des Textes im historischen Gefüge seiner Zeit hinweist: 24. Juni 1955, Paris ist der Veröffentlichung in Itinerário beigefügt. ${ }^{41}$ Die vorletzte Ausgabe der Monatszeitschrift, in der das Gedicht erschien, wird durch den Leitartikel Para uma cultura moçambicana (Für eine mosambikanische Kultur) eröffnet. In diesem Zusammenhang offenbart sich das Insistieren auf «a terra` in dem Gedicht Aqui nascemos auf das Weiterschreiben in und über das Land, als Begehren nach einer national rückgebundenen Literatur. Der erwähnte Leitartikel wünscht sich - in expliziter Anerkenntnis der heterogenen EinwohnerInnen - «uma expressão moçambiquana feita em Moçambique por moçambicanos. [...].» ${ }^{42} \mathrm{Im}$ Exil in Paris wird das national orientierte literarische Schaffen von Dos Santos jedoch gerade durch den

41 Vgl. Kalungano: Aqui nascemos.

42 Anonym: Para uma cultura moçambicana. In: ebda., S. 1. «eine mosambikanische Ausdrucksweise, die in Mosambik und für MosambikanerInnen entsteht» (meine Übersetzung). 
transnationalen, panafrikanisch orientierten Diskurs der Zeit befördert. ${ }^{43}$ Dos Santos' Schreiben ist in diesem Sinne als avantgardistisch zu verstehen, da es hier ein neues nationales Selbstbewusstsein ausdrückt, das historisch und nicht essentialistisch bestimmt ist. Es hat damit Teil an einem eigenständigen Diskurs, der sich in der zeitgenössischen mosambikanischen Lyrik verstärkt formierte. ${ }^{44}$

Besonders wertvoll für das Erkenntnisinteresse ist auch eine ebenfalls von Dos Santos' Weggefährten und Kollegen De Andrade besorgte Anthologie, ${ }^{45}$ die 1958 zuerst in Paris erschien und die das zweite Gedicht Dos Santos' enthält, das hier besprochen werden soll. Die Anthologie verweist zwar emblematisch auf die négritude, die mit Senghor in Verbindung gebracht wird: Der Titel der Anthologie Antología da poesia negra de expressão portuguesa, verweist nicht zufällig auf die zehn Jahre zuvor veröffentlichte Anthologie de la nouvelle poésie nègre et malgache de langue française von Senghor, ${ }^{46}$ die Jean-Paul Sartre mit seinem ikonischen gewordenen Vorwort Orphée noir versah. ${ }^{47}$ Jedoch setze der Herausgeber Mário

43 Mbembe bemerkt, dass sich der «Rehabilitierungsdiskurs» im 20. Jahrhundert und die Diskussionen um Nationalismus in Afrika nicht frei machten von der biologistisch fundierten Kategorie der 〈Rasse`: «Tatsächlich übernimmt er [dieser Diskurs] diese Fiktion [der Rasse und des Rassensubjektes]. Das gilt sowohl für die Négritude als auch für die verschiedenen Varianten des Panafrikanismus. [...] Die Rasse dient als Beweis (und bisweilen als Rechtfertigung) für die Existenz der Nation. Sie ist das moralische Subjekt und zugleich die dem Bewusstsein immanente Tatsache.» Es werde versucht, «eine Quasi-Äquivalenz zwischen Rasse und Geographie herzustellen und dann die kulturelle Identität aus dem Verhältnis zwischen den beiden hervorgehen zu lassen. [...] Durch die Vermengung von rassischer Authentizität und Territorialität wird Afrika zum Land der Schwarzen. [...] Der Bezug auf die Rasse bildet hier die Grundlage der staatsbürgerlichen Verwandtschaft. [...] Der Gedanke eines Afrikanertums, das nicht schwarz wäre, ist schlichtweg undenkbar.» Achille Mbembe: Kritik der schwarzen Vernunft, S. 172-175. Allerdings müsste seine Feststellung im letzten Satz mit Blick auf Mosambik differenziert werden: Malyn Newitt sagt dazu: "One notable feature is the importance of the creole element in the population [...]. Marcelino dos Santos [...] is a mestizo, while Mia Couto, the most prominent Mozambican writer [...] is of white Portuguese origin.» Malyn Newitt: $A$ short history of Mozambique, S. $218 \mathrm{f}$.

44 Die Dichterin Noémia de Sousa mag als profilstärkste Autorin in diesem Hinblick gelten. Vgl. Fátima Mendonça: Moçambique, lugar para a poesia. In: Noémia de Sousa: Sangue negro. Maputo: Associação dos Escritores Mocambicanos 1998, S. 161-174, hier S. 167.

45 Mário Pinto de Andrade (Hg.): Antologia da poesia negra de expressão portuguesa. Paris: Pierre Jean Oswald 1958.

46 Léopold Sédar Senghor (Hg.): Anthologie de la nouvelle poésie nègre et malgache de langue française. Précédée de «Orphée noir» par Jean-Paul Sartre. Paris: PUF 1948.

47 Siehe dazu die entsetzte Reaktion Fanons: «quand je lus cette page, je sentis qu'on me voulait ma dernière chance. [...] cet ami [Sartre] n'avait rien trouvé de mieux que de montrer la relativité de leur action [des peuples de couleur] [...]. J.-P. Sartre, dans cette étude, a détruit l'enthousiasme noir.» Frantz Fanon: Peau noire et masques blancs. Paris: Édition du Seuil 1952, S. 135f. Für die heutige Négritude-Diskussion im Bemühen, dem Vorwurf des Essentialismus zu begegnen vgl. 
Pinto de Andrade durch die Auswahl der Texte den Akzent auf einem négritudeVerständnis, das eher Césaire nahesteht. ${ }^{48}$ Diese Auslegung sah sich zwar auch einem panafrikanisch fokussierten, prinzipiell universellen Humanismus ${ }^{49}$ verschrieben, aber stellte vor allem die politische Unabhängigkeit der kolonisierten Länder in den Vordergrund. ${ }^{50}$ Insofern kann die Anthologie als Signum für den veränderten Umgang der SchriftstellerInnen-Generation von Dos Santos mit der négritude interpretiert werden: weg von der Herausbildung eines panafrikanischen Bewusstseins hin zur Vision unabhängiger afrikanischer Länder. ${ }^{51}$

Davon zeugt auch Marcelino Dos Santos' Gedicht Onde estou, ${ }^{52}$ das historisch dimensioniert ist: Es ist die kraftvolle Artikulation eines lyrischen Ichs in einem

Babacar Mbaye Diop: «Orphée noir»: une fausse idée sartrienne de la négritude. In: Ders. (Hg.): Le destin de la négritude. Paris: Gallimard 2009 (Editions de la lune), S. 17-26.

48 In seinem Vorwort spricht De Andrade explizit von einer Überwindung einer essentialistisch verstandenen négritude, die sich auf eine bloße Affirmation des eigenen Seins beziehe. Universalismus hieße demnach, die eigene sozio-kulturelle und historische Partikularität zu stärken. Vgl. Mário Pinto de Andrade: Préfacio. In: Ders. (Hg.): Antologia da poesia negra de expressão portuguesa, S. XIV.

49 «Wer behauptet, die Welt reduziere sich nicht auf Europa, der rehabilitiert Singularität und Differenz. Darin ist Césaire sehr nahe bei Senghor, was immer man sonst über sie sagen mag. Beide lehnen abstrakte Visionen des Universellen ab. Sie machen geltend, dass das Universelle sich stets im Register des Besonderen dekliniere.» Achille Mbembe: Kritik der schwarzen Vernunft, S. 218. Zur Frage der Universalität vgl. insb. Léopold Sédar Senghor: Liberté III. Négritude et civilisation de l'universel. Paris: Seuil 1977.

50 Pires Laranjeira fasst die grundsätzliche Akzentverschiebung bei Césaire und Senghor folgendermaßen: «Vemos, pois, uma Negritude de discurso literário agressivo, política- e ideologicamente compulsiva, tendo Césaire como farolim anti-colonial, sem contemplações para com um Ocidente vituperado até à exaberação, enquanto outra, mais compassiva e, pode-se dizer, contemplativa, se sustentava no espírito e letra senghorianos de conciliação personalista e cristã dos elementos culturais europeus e africanos, na espinhosa via do que (se) chamou a «civilização do universal.»»

(«Wir haben es sodann mit einer Negritude zu tun, die einen aggressiven literarischen Diskurs darstellt, die unbedingt politisch und ideologisch ist, und Césaire als antikolonialen Vorkämpfer hat, die ohne Beschaulichkeiten gegenüber dem Westen auskommt, den sie bis zur Erschöpfung anklagt. Die andere, weichere, und man kann sagen, beschaulichere, stützte sich auf den Geist und das Schreiben Senghors, geprägt von einer persönlichen und christlichen Versöhnung der europäischen und afrikanischen kulturellen Elemente, auf dem steinigen Weg zu erreichen, der sich «universelle Zivilisation` nannte.») (meine Übersetzung)

Pires Laranjeira (Hg.): Negritude africana de língua portuguesa: textos de apoio (1947-1963). Braga: Angelus Novus 2001, S. XXI. Vgl. grundsätzlich zur lusophonen négritude dessen ausführliche Dissertation: Pires Laranjeira: A negritude africana de língua portuguesa. Tese de doutoramento em Literaturas dos países africanos de expressão portuguesa. Coimbra: Universidade de Coimbra 1994.

51 Vgl. Fátima Mendonça: O entrelugar da escrita, S. 97-108.

52 Vgl. Marcelino dos Santos: Onde estou. In: Ders.: Canto do amor natural, ab S. 35. 
rassistisch-kolonial geprägten Umfeld. Im Gegensatz zu dem ersten hier besprochenen Text verortet sich das lyrische Ich innerhalb einer intellektuellen, diskursiven Topographie. Damit schließt es sich einer Interpretation von négritude an, die Césaire in einer späteren Rede folgendermaßen gefasst hat:

C'est une manière de vivre l'histoire dans l'histoire - l'histoire d'une communauté dont l'expérience apparaît, à vrai dire, singulière avec ses déportations de populations, ses transferts d'hommes d'un continent à l'autre, les souvenirs de croyances lointaines, ses débris de cultures assassinées. Comment ne pas croire que tout cela qui a sa cohérence constitue un patrimoine $?^{53}$

Das Gedicht Onde estou knüpft an einen prominenten Text im lusophonen Umfeld der négritude an: Negro de tudo o mundo von Francisco José Tenreiro ${ }^{54}$ aus dem westafrikanischen Inselstaat São Tomé e Príncipe: ${ }^{55}$

\begin{tabular}{ll}
\hline Onde estou & Wo ich bin \\
Não & Nein \\
$\begin{array}{l}\text { Não me procureis } \\
\text { onde não existo }\end{array}$ & $\begin{array}{l}\text { Sucht mich nicht } \\
\text { wo ich nicht existiere }\end{array}$ \\
$\begin{array}{l}\text { Eu vivo } \\
\text { curvado sobre a terra } \\
\text { seguindo o caminho inscrito }\end{array}$ & $\begin{array}{l}\text { Ich lebe } \\
\text { gekrümmt über der Erde } \\
\text { ich folge dem Weg eingeschrieben }\end{array}$ \\
\hline
\end{tabular}

53 Aimé Césaire: Discours sur la négritude. Discours prononcé le jeudi 26 février 1987 à l'Université internationale de Floride à Miami. In: Ders.: Discours sur le colonialisme. Paris: Présence Africaine 2004, S. 82.

54 Vgl. Francisco José Tenreiro: Negro de todo o mundo. In: Ders.: Ilha de nome santo. Coleção Novo Cancioneiro. Coimbra: Portugália 1942, S. 37-40.

55 Tenreiro war beim portugiesischen Kolonialministerium beschäftigt und setzte in Lissabon unter den afrikanischen Studierenden maßgebliche Impulse für die künstlerische Arbeit, wie sich Mário Pinto de Andrade, mit dem er ebenfalls herausgeberisch tätig war (vgl. Anm. 58), sich erinnert. Vgl. Michel Laban: Uma entrevista, S. 62f.

56 Zitiert wird hier nach Marcelino dos Santos: Canto do amor natural, S. 35-37. Die Anthologie von 1958 konnte leider für den vorliegenden Beitrag nicht im Detail eingesehen werden. Möglicherweise gibt es Abweichungen in der Versgestaltung. Dass es sich im Großen und Ganzen um die gleiche Version des Gedichtes handelt, legt die Übersetzung ins Französische nahe, die von dem Dichter René Depestre besorgt wurde. Dort ist als Autor Kalungano angegeben. Vgl. René Depestre/Kalungano: Ou suis-je? In: Présence Africaine 20 (Juni-Juli 1958), S. 62-63. Die zeitnahe Übersetzung ins Französische und die Publikation im populärsten Medium für panafrikanische Kurse jener Zeit belegt, dass Marcelino dos Santos von seinen Zeitgenossen durchaus als wichtiger Autor für die Debatten über die négritude wahrgenommen wurde. 


\begin{tabular}{|c|c|}
\hline $\begin{array}{c}\text { pelo chicote } \\
\text { nas minhas costas nuas }\end{array}$ & $\begin{array}{c}\text { von der Peitsche } \\
\text { in meinen nackten Rücken }\end{array}$ \\
\hline $\begin{array}{l}\text { Eu vivo } \\
\text { nos portos } \\
\text { alimentando as fornalhas } \\
\text { movendo as máquinas } \\
\text { pelo caminho dos homens }\end{array}$ & $\begin{array}{l}\text { Ich lebe } \\
\text { in den Häfen } \\
\text { ich befeure die Öfen } \\
\text { ich bewege die Maschinen } \\
\text { auf dem Weg der Menschen }\end{array}$ \\
\hline $\begin{array}{l}\text { Eu vivo } \\
\text { no corpo de minha mãe } \\
\text { vendendo a minha carne } \\
\quad \text { o meu sexo } \\
\quad \text { não é para amar }\end{array}$ & $\begin{array}{l}\text { Ich lebe } \\
\text { im Körper meiner Mutter } \\
\text { ich verkaufe mein Fleisch } \\
\text { mein Geschlecht } \\
\text { ist nicht für die Liebe }\end{array}$ \\
\hline $\begin{array}{l}\text { Eu vivo } \\
\text { perdido nas ruas } \\
\text { de uma civilização } \\
\text { que me esmaga } \\
\text { com ódio } \\
\text { sem pena }\end{array}$ & $\begin{array}{l}\text { Ich lebe } \\
\text { verloren in den Straßen } \\
\text { einer Zivilisation } \\
\text { die mich erdrückt } \\
\text { mit Hass } \\
\quad \text { ohne Mitleid }\end{array}$ \\
\hline $\begin{array}{l}\text { E se é a minha voz que se ouve } \\
\text { e se sou eu que canto ainda } \\
\text { é porque não posso morrer } \\
\text { mas só a lua escuta } \\
\text { a minha dor }\end{array}$ & $\begin{array}{l}\text { Und wenn es meine Stimme ist, die man hört } \\
\text { und wenn ich es bin, der noch immer singt } \\
\text { dann ist es, weil ich nicht sterben kann } \\
\text { aber nur der Mond hört } \\
\text { meinem Schmerz zu }\end{array}$ \\
\hline Não & Nein \\
\hline $\begin{array}{l}\text { não me procureis } \\
\text { nos grande salões } \\
\text { onde não estou } \\
\text { onde não posso } \\
\text { estar }\end{array}$ & $\begin{array}{l}\text { sucht mich nicht } \\
\text { in den großen Salons } \\
\text { wo ich nicht bin } \\
\text { wo ich nicht sein } \\
\text { kann }\end{array}$ \\
\hline $\begin{array}{l}\text { Aqui na América } \\
\text { sim } \\
\text { eu estou também } \\
\text { eu estou }\end{array}$ & $\begin{array}{l}\text { Hier in Amerika } \\
\text { ja } \\
\text { hier bin ich auch } \\
\text { hier bin ich }\end{array}$ \\
\hline $\begin{array}{l}\text { Mas Lincoln } \\
\text { foi assassinado }\end{array}$ & $\begin{array}{l}\text { Aber Lincoln } \\
\text { wurde ermordet }\end{array}$ \\
\hline $\begin{array}{l}\text { e eu } \\
\text { eu }\end{array}$ & $\begin{array}{l}\text { und ich } \\
\text { ich } \\
\\
\end{array}$ \\
\hline
\end{tabular}




\begin{tabular}{|c|c|}
\hline todos os dias sou linchado & jeden Tag werde ich gelyncht \\
\hline 0 comboio especial & Der Sonderzug \\
\hline rolando vertiginosamente na estrada & rollt schwindelerregend auf der Straße \\
\hline é ouro & es ist Gold \\
\hline é sangue & es ist Blut \\
\hline que eu verti através dos séculos & das ich vergossen habe über die Jahrhunderte hinweg \\
\hline Porquê & Warum \\
\hline pois & also \\
\hline procurar-me na Glória de Beethoven & mich suchen im Gloria Beethovens \\
\hline se eu estou aqui & wenn ich hier bin \\
\hline erguendo-me & mich aufrichte \\
\hline nos milhões de ais & in den Millionen von Schmerzensrufen \\
\hline que se elevam dos porões & die aus den Laderäumen steigen \\
\hline em todos os cais & in allen Kais \\
\hline se eu estou aqui & wenn ich hier bin \\
\hline bem vivo & sehr lebendig \\
\hline na voz de Robeson e Hughes & in der Stimme der wiedergeborenen Robeson und Hughes \\
\hline Césaire e Guillén & Césaire und Guillén \\
\hline Godido e Black Boy renascidos & Godido und Black Boy \\
\hline nas entranhas da terra & in den Eingeweiden der Erde \\
\hline transformando com o meu corpo & mit meinem Körper verwandle ich \\
\hline os alicerces da vida & die Fundamente des Lebens \\
\hline se eu estou aqui & wenn ich hier bin \\
\hline soma consciente e firme & bewusstes und kraftvolles Ergebnis \\
\hline dos homens & der Menschen \\
\hline que compuseram o poema & die das Gedicht komponiert haben \\
\hline da vida contra a morte & vom Leben gegen den Tod \\
\hline do fim da noite & vom Ende der Nacht \\
\hline e do começo da dia & und dem Beginn des Tages \\
\hline
\end{tabular}

Das Gedicht Onde estou ist in freien Versen gehalten, die zum Teil graphisch durch Einrücken abgesetzt sind. Bereits in der zweiten Zeile wendet sich das lyrische Ich an ein lyrisches Du. Auf die negativ formulierten Imperative in den Versen eins bis drei antworten vier affirmative Strophen, die jeweils mit dem Vers «Eu vivo〉 beginnen. Jede der Strophen ist durch eine Spannung gekennzeichnet: 
Die Bekräftigung des lyrischen Ichs 〈Eu vivo〉 ist prioritär, steht es doch unumstößlich vor der Beschreibung schwieriger durch Unterdrückung, Marginalisierung und Gewalt geprägter Lebenssituationen: Zunächst die Zwangsarbeit, die durch die Peitsche in die menschlichen Körper gleichsam eingeschrieben wird (vgl. V. 5-8); dann Hafenarbeit (vgl. V. 10-13) und Prostitution (vgl. V. 16-18). Die Verse 20-24 umreißen die doppelte, scheinheilige Moral der angeblich Fortschritt bringenden, jedoch rassistisch und hassmotivierten Unternehmung des Kolonialismus: Das lyrische Ich ist «perdido nas ruas de uma civilização», wobei das Wort «Zivilisation` ironischen Klang annimmt, wenn es mit abschließender Antithese weiter heißt: «que me esmaga / com ódio / sem pena».

Zum Schluss der ersten Hälfte des Gedichtes evoziert das lyrische Ich mit der Formulierung «E se é a minha voz que se ouve / e se sou eu que canto ainda» (V. 25f.) die Dichtung, die trotz allem nicht zum Schweigen zu bringen ist. «Não posso morrer» (V. 26) gibt das lyrische Ich noch an. An mangelndem Vermögen scheint es nicht zu liegen, heißt es doch nicht «não consigo - oder - não sei morrer». Die Stimme kann wohl nicht sterben, weil beziehungsweise solange sie Teil von so vielen anderen, solidarisch gesinnten ist, wie die zweite Hälfte des Gedichtes thematisiert. Diese beginnt mit einer Wiederholung der ersten Verse und gibt im Kontrast dazu an, wo das lyrisch Ich nicht sein kann ${ }^{57}$, «nos grandes salões» (V. 31). Die Sphären der bürgerlichen Elite bleiben dem lyrischen Ich verschlossen. In Vers 35 verortet die hic-et-nunc-Deixis das lyrische Ich in Amerika, womit auf die durch den Sklavenhandel geprägte Beziehung zwischen Afrika und dem amerikanischen Kontinent verwiesen wird. Amerika kann außerdem als Chiffre für die nordamerikanische Bewegung der Harlem Renaissance sowie des lateinamerikanischen negrismo gelesen werden. ${ }^{58}$ Beides sind Momente, die die afrikanischen DichterInnen stark beeinflussten - zahlreiche Verweise ziehen sich beispielsweise durch das Werk Noémia de Sousas. ${ }^{59}$ Die Ermordung des amerikanischen Präsidenten Lincoln, der die Versklavung rechtlich abschaffte, wird im Gedicht verbunden mit dem alltäglichen Sterben afrikanischer Menschen. Das dreifach wiederholte und graphisch in Stufenform angeordnete Personalpronomen «eu

57 Vgl. zum Topos des Nicht-dort-Seins Achille Mbembe: Kritik der schwarzen Vernunft, S. 64, insb. Anmerkung 73.

58 Mit Blick auf Verknüpfung der verschiedenen Diskurse in der afrikanischen und afroamerikanischen Sphäre nennt Souleymane Bachir Diagne die Négritude «cet enfant francophone de la Harlem Renaissance.» Souleymane Bachir Diagne: Éloge du postracial: La négritude au-delà de la négritude. Préface. In: Babacar Mbaye Diop (Hg.).: Le destin de la négritude. Paris: Gallimard 2009 (Editions de la lune), S. 9-16, hier S. 9.

59 Vgl. Noémia de Sousa: Sangue negro. Maputo: Associação dos Escritores Mocambicanos 1998. 
unterstreicht die anhaltende Gewalt (vgl. V. 41-44). Genannt sei an dieser Stelle beispielsweise das Massaker in Batepá auf São Tomé, 1953, bei dem Angehörige der portugiesischen Kolonialverwaltung sowie portugiesische LandbesitzerInnen hunderte Einheimische ermordeten. ${ }^{60}$ Das lyrische Ich markiert nachdrücklich seine Solidarität, stirbt es doch mit jedem mit, der umgebracht wird. ${ }^{61}$

Das lyrische Ich, das sich - wie gesehen - in kollektiver Dimension versteht, ist nicht in europäischen Kunsterzeugnissen $\mathrm{zu}$ finden, etwa nicht bei Beethoven (vgl. V. 50-53). Vielmehr fühlt es sich - im Sinne der négritude - «eigenen» kulturellen Äußerungen nahe: Robeson und Hughes verweisen einmal mehr auf die Harlem Renaissance, die Anfang des 20. Jahrhunderts afrikanische Kunst, Kultur und Geschichte zur Geltung bringen wollte. Die in dem Gedicht erwähnten Dichter Nicolàs Guillén und Aimé Césaire können als geistige Paten zweier verschiedener Generationen der anti-essentialistisch akzentuierten Selbstbekundung in der Literatur verstanden werden. Diese findet Ausdruck in den jeweils spezifisch kulturell-historischen Konfigurationen des negrismo im hispanoamerikanischen und der négritude im französischsprachigen Raum. ${ }^{62}$ Mit dem Namen Guillén ist weiterhin die Erinnerung an die erste, prominente Gedichtsammlung mosambikanischer AutorInnen von 1953 - in der Dos Santos allerdings noch nicht auftaucht - aufgerufen. Der Herausgeber De Andrade widmete das Carnet, das als Vorläufer der in Paris erschienen Anthologie von 1958 gelten kann, ausdrücklich

60 Vgl. Gerhard Seibert: Le massacre de février 1953 à São Tomé. Raison d'être du nationalisme santoméen. In: Lusotopie (1997), S. 173-192.

61 Es wird in dem Gedicht nicht explizit gemacht, auf welche historischen Gewaltereignisse Bezug genommen wird. Batepá wäre eine Möglichkeit, denn Mário Pinto de Andrade wusste vergleichsweise früh darüber und schrieb einen Artikel für die Zeitschrift Présence Africaine 1955. Vgl. ebda., S. 173. Ich danke Alexander Keese von der Universität Genf für diesen Hinweis.

62 Zum Verhältnis von Guillén und Césaire halten Gisela Felbel und Natascha Ueckmann fest: «Zweierlei ist hier bemerkenswert: Zum einen liegen die Ursprünge dieser besonderen Reflexion über Identität und Alterität geographisch nicht in der Metropole wie bei der Négritude, sondern von Anfang an in der «Peripheries. Zum anderen ist Guilléns Ausgangspunkt kein universelles Schwarz-Sein, sondern er setzt an den Anfang das Gemischte, das komplex miteinander Verwobene und Vermengte von afrikanischen Ursprüngen und europäisch-kolonialen Einflüssen, wie es in der Frankokaribik erst später im Éloge de la Créolité oder bei Édouard Glissant formuliert wird. Aber genau diese «plurietnicidad autorial de facto` (Branche 1999: 484) ist für Guillén das Kubanische, das sich in der Lebensweise, der Musik, dem Son, und nicht in der Hautfarbe oder in einem definierten Ursprung ausdrückt. Er hat insofern bereits eine anti-essentialistische Position, die auch der Vereinnahmung durch einen europäischen Exotismus in der Rezeption einen gewissen Widerstand leistet [...].» Natascha Ueckmann/Gisela Felbel: Négritude und Negrismo afrokaribische Literaturen und neue Humanismen. In: Dies. (Hg.): Pluraler Humanismus. «Négritude» und «Negrismo» weitergedacht. Berlin: Springer 2017, S. 9-53, hier S. 24. 
dem kubanischen Dichter, «a voz mais alta da negritude de expressão hispanoamericana.» ${ }^{63}$ Die Nennung Césaires - und nicht Senghors - spezifiziert darüber hinaus, dass sich das Gedicht in den Kontext seines négritude-Verständnisses stellt und nicht in den eines «racisme anti-raciste», wie es Sartre im Vorwort zu Senghors berühmt gewordener Anthologie formuliert hatte. ${ }^{64}$

Die Verse 59-66 bilden mit Abstand die längste und durch die Dichte der Verweise auch die kompakteste Strophe. Die Wiederholung der Phrase «se eu estou aqui» (vgl. V. 53, 58 und schließlich V. 66) strukturiert diesen letzten Teil des Gedichtes, indem die Präsenz des lyrischen Ichs geradezu triumphiert. Besonders bemerkenswert sind die Erwähnungen der Namen Godido und Black Boy in Vers 62, denn sie situieren das Gedicht in einer autoreflexiven Geste - sprachlich angezeigt durch die wiederaufgenommene Vokabel 〈voz〉 aus der selbstbezüglichen, bereits analysierten Passage zu Beginn des Gedichtes - in einem weiten Netz spezifisch afrikanischer intertextueller und kultureller Bezüge. Die Referenz auf den Namen Godido hat mehrere Implikationen: Einerseits stellt sie den Bezug zur historischen Figur her, nämlich zum Sohn des letzten Herrschers des historischen Staates Gaza ${ }^{65}$ (1824-1895) im heutigen Mosambik, Simbabwe und Südafrika, der den Portugiesen Widerstand leistete und sich 1895 schließlich geschlagen geben musste. Andererseits ist sie Berufung auf ein gemeinsames kollektives Gedächtnis sowie intertextueller Verweis: João Dias ${ }^{\text {,66 }}$ Werk Godido $e$ outros contos, das posthum 1952 in der Serie Autores Últramarinos der CEI erschien, gilt als eines der ersten Werke portugiesischsprachiger Schriftliteratur aus Mosambik. Außerdem figurierte der Bezug zu Godido in vielen Texten mosambikanischer DichterInnen der Zeit, wie beispielsweise im gleichnamigen Gedicht Noémia de Sousas. Black Boy wiederum ist der Titel der 1945 erschienenen

63 Mário Pinto de Andrade/Francisco José Tenreiro (Hg.): Caderno de poesia negra de expressão portuguesa. Mit einem Vorwort von De Andrade und einem Schlusswort von Tenreiro. Lissabon: Casa dos Estudantes do Império 1953, S. 4. In der knappen Zusammenstellung sind Gedichte vertreten von Nicolas Guillén, Alda do Espírito Santo, Agostinho Neto, António Jacinto, Francisco José Tenreiro, Noémia de Sousa, Viriato da Cruz und Orlando da Costa.

64 Vgl. dazu auch den Beitrag von Ibou Coulibaly Diop im vorliegenden Sammelband. Bei der größeren Affinität zu Césaire spielt dessen Angehörigkeit zur Kommunistischen Partei - zumindest bis eben 1956 - sicher eine Rolle, war Senghor doch ein katholischer Sozialdemokrat. Vgl. Pires Laranjeira: A negritude africana de língua portuguesa, S. 346.

65 Vgl. Petar Petrov: O projecto literário de Mia Couto. Lissabon: CLEPUL/LusoSofia Press 2014, S. 8.

66 Vgl. João Dias. In: Artigos de apoio Infopédia. Porto: Porto Editora 2003-2017. <https://www. infopedia.pt/apoio/artigos/\$joao-dias> [04.10.2017]. 
Autobiographie des US-Schriftstellers Richard Wright, ${ }^{67}$ der ebenfalls am Schriftstellerkongress 1956 in Paris teilnahm.

Das Gedicht Onde estou schließt auf einem optimistischen, kämpferischen Akkord. Das lyrische Ich, das sich im Sprechen und im Dialog mit den solidarischen Stimmen aus der vorhergehenden Strophe realisiert, begreift sich als vitale Kraft des Widerstandes: «soma consciente e firme / dos homens / que compuseram o poema / da vida contra a morte / do fim da noite / e do começo da dia» (V. 67-72).

Im Hinblick auf Dos Santos’ Dichtung lässt sich sagen, dass seine avantgardistische Praxis im Paris der 1950er Jahre mehr im Gestus des Schreibens als solchem liegt als in hyperkomplexen formal-ästhetischen Verfahren. Seine Gedichte haben Teil an der Mitbegründung portugiesischsprachiger Schriftliteratur aus Mosambik. Im Modus des Lyrischen artikulieren die Texte ein neues Selbstbewusstsein in der ersten Person Singular. Die Kulturschaffenden der Zeit erheben Anspruch auf diesen innovativen Charakter und bringen die literarische Produktion ihrer Landsleute dezidiert in Verbindung $\mathrm{zu}$ diesem, immer politisch antikolonial orientierten, Diskurs.

\section{Literaturverzeichnis}

Anonym: Para uma cultura moçambicana. In: Itinerário 149 (Juli/August 1955), S. 1.

Andrade, Mário Pinto de (Hg.): Antologia temática de poesia africana 2. 0 canto armado. Lissabon: Sá da Costa 1979.

Andrade, Mário Pinto de (Hg.): Antologia temática de poesia africana 1. Na nojte grávida de punhais. Lissabon: Sá da Costa 1975.

Andrade, Mário Pinto de (Hg.): Antologia da poesia negra de expressão portuguesa. Paris: Pierre Jean Oswald 1958.

Andrade, Mário Pinto de/Francisco José Tenreiro (Hg.): Caderno de poesia negra de expressão portuguesa. Mit einem Vorwort von De Andrade und einem Schlusswort von Tenreiro. Lissabon: Casa dos Estudantes do Império 1953.

Archer-Straw, Petrine: Avant-Garde Paris and Black Culture in the 1920s. New York u. a.: Thames and Hudson 2000.

Césaire, Aimé: Discours sur la négritude. Discours prononcé le jeudi 26 février 1987 à l'Université internationale de Floride à Miami. In: Ders.: Discours sur le colonialisme. Paris: Présence Africaine 2004.

Césaire, Aimé: Lettre à Maurice Thorez. Paris: Présence Africaine 1956.

Chabal, Patrick: The Postcolonial Literature of Lusophone Africa. Evanston: Northwestern University Press 1996 (African Studies).

Depestre, René: Bonjour et adieu à la négritude. Paris: Robert Laffont 1980.

67 Richard Wright: Black Boy. New York: Harper and Brothers 1945. 
Depestre, René/Kalungano [Marcelino dos Santos]: Ou suis-je?. In: Présence Africaine 20, Nouvelle Série (Juni-Juli 1958), S. 62-63.

Diagne, Souleymane Bachir: Éloge du postracial: La négritude au-delà de la négritude. Préface. In: Babacar Mbaye Diop (Hg.).: Le destin de la négritude. Paris: Gallimard 2009 (Editions de la lune), S. 9-16.

Diop, Alioune: Discours d’ouverture. In: Présence Africaine 8/10 (Juni-November 1956),

S. 9-19.

Diop, Alioune: Niam n'goura : ou les raisons d'être de Présence Africaine. In: Présence Africaine 1 (November-Dezember 1947), S. 7-14.

Diop, Babacar Mbaye: 〈Orphée noir` : une fausse idée sartrienne de la « négritude ». In: Ders.

(Hg.): Le destin de la négritude. Paris: Gallimard 2009 (Editions de la lune), S. 17-26.

Eckert, Andreas: Universitäten und die Politik des Exils. Afrikanische Studenten und anti-koloniale Politik in Europa, 1900-1960. In: Rüdiger vom Bruch u. a. (Hg.): Jahrbuch für Universitätsgeschichte 7. Stuttgart: Franz Steiner 2004, S. 129-145.

Fanon, Frantz: Peau noire et masques blancs. Paris: Édition du Seuil 1952.

Greene, Roland/Stephen Cushman u. a. (Hg.): The Princeton Encyclopedia of Poetry and Poetics. Princeton: Princeton University Press ${ }^{4} 2012$.

Helgesson, Stefan: Shifting Fields: Imagining Literary Renewal in Itinerário and Drum. In: Research in African Literatures 38, 2 (Sommer 2007), S. 206-226.

Helgesson, Stefan: In search of newness. In: Scrutiny2. Issues in English Studies in Southern Africa 10, 2 (2005), S. 15-23.

Jules-Rosette, Bennetta: Black Paris. The African Writers' Landscape. Urbana/Chicago: University of Illinois Press 1998.

Kalungano [Marcelino dos Santos]: Aqui nascemos. In: Itinerário 149 (Juli/August 1955), S. 13. Kesteloot, Lylian: Césaire et Senghor. Un pont sur l'Atlantique. Paris: L'Harmattan 2006. Laban, Michel: Mário Pinto de Andrade: uma entrevista dada a Michel Laban. Übersetzung von Maria Alexandra Dáskalos. Lissabon: João Sá da Costa 1997.

Laranjeira, Pires (Hg.): Negritude africana de língua portuguesa: textos de apoio (1947-1963). Braga: Angelus Novus 2001.

Laranjeira, Pires: A negritude africana de língua portuguesa. Tese de doutoramento em Literaturas dos países africanos de expressão portuguesa. Coimbra: Universidade de Coimbra 1994.

Lipphardt, Anna: Der Nomade als Theoriefigur, empirische Anrufung und Lifestyle-Emblem. Auf Spurensuche im Globalen Norden. In: Aus Politik und Zeitgeschichte 65, 26-27 (2015), S. 32-38.

Mata, Inocência: A Casa dos Estudantes do Império e o lugar da literatura na consciencialização política. Lissabon: União das Cidades Capitais de Língua Portuguesa 2015.

Mbembe, Achille: Kritik der schwarzen Vernunft. Aus dem Französischen von Michael Bischoff. Berlin: Suhrkamp 2017 (Taschenbuch Wissenschaft 2205).

Mendonça, Fátima: 0 entrelugar da escrita: entre Marcelino ideólogo e Kalungano poeta. In: Dies.: Literatura Moçambicana - as dobras da escrita. Maputo: Ndjira 2011, S. 97-108.

Mendonça, Fátima: Moçambique, lugar para a poesia. In: Noémia de Sousa: Sangue negro. Maputo: Associação dos Escritores Mocambicanos 1998, S. 161-174.

Newitt, Malyn: A short history of Mozambique. London: Hurst and Company 2017.

Ohne Autor: João Dias. In: Artigos de apoio Infopédia. Porto: Porto Editora 2003-2017. <https://www.infopedia.pt/apoio/artigos/\$joao-dias> [04.10.2017].

Petrov, Petar: O projecto literário de Mia Couto. Lissabon: CLEPUL/LusoSofia Press 2014. 
Rosário Rosinha, Maria do (Hg.): Antologias de poesia da Casa dos Estudantes do Império 19511963. Bd. 2. Mosambik/Lissabon: União das Cidades Capitais de Língua Portuguesa, 2014. Rose, Phyllis: Jazz Cleopatra: Josephine Baker in her Time. New York: Vintage 1991.

Santos, Marcelino dos: Canto do amor natural. Maputo: Associação dos Escritores Moçambicanos 1984.

Seibert, Gerhard: Le massacre de février 1953 à São Tomé. Raison d'être du nationalisme santoméen. In: Lusotopie 1997, S. 173-192.

Senghor, Léopold Sédar: Liberté III. Négritude et civilisation de l'universel. Paris: Seuil 1977.

Senghor, Léopold Sédar (Hg.): Anthologie de la nouvelle poésie nègre et malgache de langue française. Paris: PUF 1948.

Sousa, Noémia de: Sangue negro. Maputo: Associação dos Escritores Mocambicanos 1998.

Tenreiro, Francisco José: Negro de todo o mundo. In: Ders.: Ilha de nome santo. Coleção Novo Cancioneiro. Coimbra: Portugália 1942, S. 37-40.

Ueckmann, Natascha/Gisela Felbel: Négritude und Negrismo - afrokaribische Literaturen und neue Humanismen. In: Dies. (Hg.): Pluraler Humanismus. «Négritude» und «Negrismo» weitergedacht. Berlin: Springer 2017, S. 9-53.

Wright, Richard: Black Boy. New York: Harper and Brothers 1945. 